AMSE JOURNALS-AMSE IIETA publication-2017-Series: Advances C; Vol. 72; $N^{\circ} 1$; pp 25-47

Submitted Jan. 2017; Revised March 15, 2017, Accepted April 15, 2017

https://doi.org/10.18280/ama_c.720103

\title{
Building and Analyzing of an Active Robot-assisted Surgical Navigation for Cervical Vertebra Bone Grinding in Spine Surgery
}

\author{
Heqiang Tia*, Longxin Ma*, Xiaoqing Dang **, Jinfeng Zhang *, Junlin Ma*** \\ * Mechanical and Electronic Engineering College, Shandong University of Science and Technology, \\ Qingdao 266590, China (Corresponding author: thq_1980@126.com) \\ **Qingdao Municipal Hospital, Qingdao266073, China \\ ***State Key Laboratory of Robotics and System, Harbin Institute of Technology, Harbin 150080, \\ China
}

\begin{abstract}
Surgical navigation has emerged as a potential solution to improve accuracy and security of intraoperative surgery. This paper introduces surgical navigation technologies into cervical vertebra bone grinding in spine surgery to guide a bone-grinding robot. Firstly, an active robot-assisted surgical navigation system based on optical positioning for cervical vertebra bone grinding is created, and the space registration coordinate system among "image-patient-robot" is also established. Secondly, space registration among “image-patient-robot" is studied. Here, intraoperative registration between image and patient is achieved by ICP improved algorithm based on coordinate system direction fitting, and space transformation between patient and robot is established by using an optical locator based on planimetric method. At last, the navigating and positioning precision of robot-assisted spine surgery is analyzed by performing positioning and tracking experiments and the influencing factors for surgical navigation errors are also analyzed. The experiment results show that the active surgical navigation system can achieve positioning and tracking of bone-grinding robot feasibly and rationally.
\end{abstract}

\section{Keywords}


Bone-grinding robot, surgical navigation, registration, tracking

\section{Introduction}

In orthopedic surgery, there is demand for a surgical tool that can be manipulated accurately and stably to the expected position/orientation on a target, e.g., to target on bone during bone resection. However, the manual procedure is dependent on surgeons' experience, and the surgery itself is far from optimum surgical plan. With recent developments in computer navigation, computer navigation systems can be adopted to obtain more accurate operation outcome by surgical planning and simulation before implementation with the assistance of surgical robotics [1-3]. Under computer navigation guidance, surgeons are now able to remove a correct shape and place cutting prosthesis in the right position and orientation.

In recent years, robot technology is widely applicable for orthopedic surgery. Thus, orthopedic procedure has become the major application field of medical robot technology [4, 5]. The combination of surgical navigation technology and robot technology expand the application space of robot technology in medical field. The application of such robot technology will fundamentally change the state of surgery operations in the 21 st century, just like robots changing the industrial processes, and promoting the rapid development of modern minimally invasive medical treatment. ROBODOC robot system [6] is a comprehensive system composed of computer-assisted medical image processing and medical robots. With the assistance of image navigation, total hip arthroplasty and total knee arthroplasty (THA \& TKA) as well as revision total knee arthroplasty (RTKA) can be conducted. The British Imperial College developed ACROBOT robot system [7] for total knee replacement and minimally invasive knee single condyle replacement, whose navigation technology is similar to ROBODOC. With the tracking and display systems, RIO (Robotic Arm Interactive Orthopaedic) system completes the bone grinding with mechanical arm during the knee arthroplasty [8]. SpineAssist [9] system is especially applicable for the spine surgery. With the intraoperative registration through CT navigation system, the robot automatically moves to the position planned; and doctor can complete the surgery as per the planned pose with the navigation arm on the robot. PinTrace is a robot-assisted multi-purpose orthopedic surgery platform, which combines the robot technology and advanced image processing technology to implement the robot surgical navigation based on intraoperative X-ray images [10]. The robot system [11] based on biplanar fluoroscopic 
navigation developed by Korean Hanyang University is used to assist the spinal fusion surgery system. Moreover, a set of biplanar robot navigation system [12] developed by Robotics Institute of Beihang University solves the problem of completing intramedullary nail distal hole locking surgery by robot navigated by X-ray images. Robotics Institute of Harbin Institute of Technology has also developed a set of robot-assisted orthopedic surgery system [13], which can guide a Motoman industrial robot to assist doctor to complete the intramedullary nail locking. Navigation systems of the surgical robots above can't actively track intraoperative surgical instruments. After the calibration between surgical image and space by outside landmarks or landmarks with optical properties, the passive navigation system no longer measures relative position between surgical equipments and bone structures in the operation process, just as a kind of intraoperative information system, which will not affect the doctor's operation. But the passive navigation system can't obtain absolute position relationship between robot and patient.

We will introduce robotics and surgical navigation technology into artificial cervical disc replacement surgery, and the positioning and tracking of robot-assisted bone-grinding is achieved with the help of active surgical navigation based on optical positioning. In this paper we present the development of an active robot-assisted navigation system based on optical positioning to assist doctors to complete complicated cervical vertebra bone grinding in spine surgery which needs accurate positioning. Here, we will overview the specific aims of our research as follows:

(1) Development of an active navigation system based on optical positioning for robot-assisted spine surgery which can help bone-grinding robot perform positioning and tracking in cervical disc replacement within limited space with minimal exposure and tissue distraction.

(2) Study of space registration among "image-patient-robot", which can achieve coordinate transformation in three spaces and positioning and tracking of bone-grinding robot.

(3) Precision analysis of navigating and positioning of robot-assisted spine surgery by performing positioning and tracking experiments so as to explore problems bone-grinding robot is facing in spine surgery.

\section{Building of active surgical navigation system}

In order to solve issues such as complicated operation and low precision of grinding equipments during the artificial cervical disc replacement surgery, the robot will automatically complete the 
grinding prosthesis cavity. How to accurately grind out prosthesis cavity depends on surgical positioning accuracy of the bone-grinding robot. In this paper, an active robot-assisted surgical navigation system based on optical positioning for cervical vertebra bone grinding is built in Fig. 1, which includes imaging device, graphic workstation, optical tracking \& positioning system, robot control computer, robot controller and surgery assisting robot. The imaging device CT is used to collect preoperative data of patient. By running navigation software, the graphic workstation mainly conduct 3D modeling and visual processing of slice sequence data collected, develop surgical planning and conduct intraoperative registration and navigation. Except for space registration, the optical tracking \& positioning system can also real-time track spatial position information of mark point on robot end-effector and conduct real-time update and visualization of such information in image space. The host control computer for robot motion-control computer mainly runs the robot control software and provides the robot-control human-computer interaction UI. The robot controller is mainly used for achieving the bottom driving and control of robot. Moreover, the surgery assisting robot is the actuator completing bone-grinding operation tasks in the whole system. With 3D image navigation technology, the system can conduct precise measurement and surgical planning in 3D space and obtain more rich surgical information than such information manually obtained with 2D CT images or X-ray films so that the surgery preparation will be more detailed. As the optical positioning technology is convenient to use and has high positioning precision, the introduction of such technology into spine surgery will change the state of complex operation and low precision of traditional mechanical positioning mode so as to improve the positioning precision of surgical instruments.

Based on requirements of spine surgery, the position \& pose of bone-grinding robot endeffector determined in image space must be transformed to the operation space of bone-grinding robot so that the bone-grinding robot can be guided to be at grinding position \& pose required by the surgical instrument, that is to establish the space registration relation among "image-patient-robot". Based on the composition of robot-assisted surgical navigation system for spine surgery and role of each part, the coordinate system of surgical navigation system can be defined and is shown in Fig. 2. Establish a model coordinate system $\{V\}$ in the computer image space; establish a patient coordinate system $\{P\}$ at the surgery spot of patient; establish a robot base coordinate system $\{R\}$ on the robot; and establish an optical measurement coordinate system $\{M\}$ on the optical locator. The patient 
coordinate system $\{P\}$ is determined by a reference passive rigid body which has its own coordinate system $\{S\}$. Besides, the bone-grinding robot end-effector with installed a passive rigid body can be defined as tracking tool coordinate system $\{T\}$.With the optical measurement coordinate system $\{M\}$ as reference system; the whole system can achieve the space transformations of each coordinate system with the optical positioning technology.

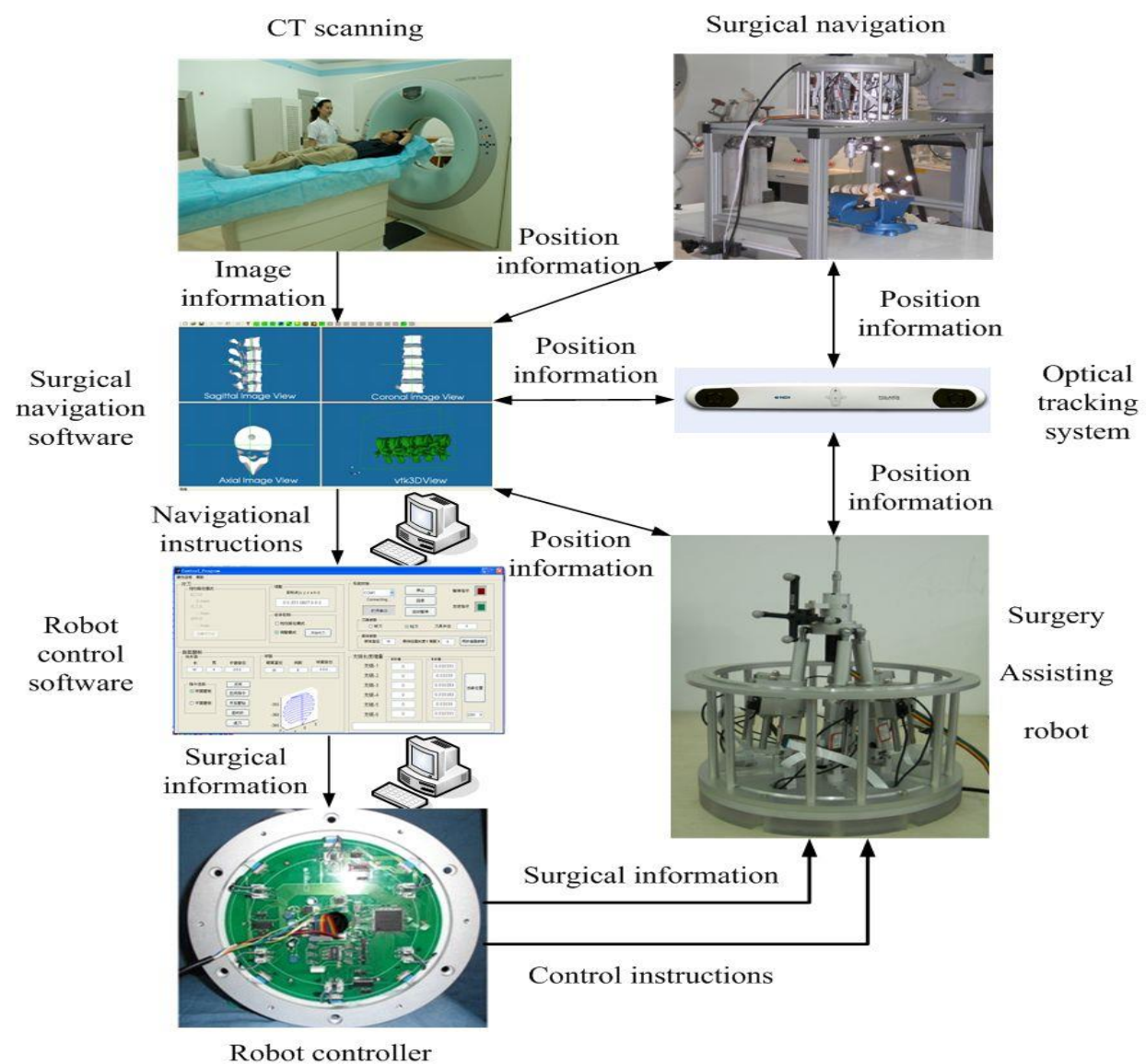

Fig. 1. Active robot-assisted surgical navigation system for cervical vertebra bone grinding 


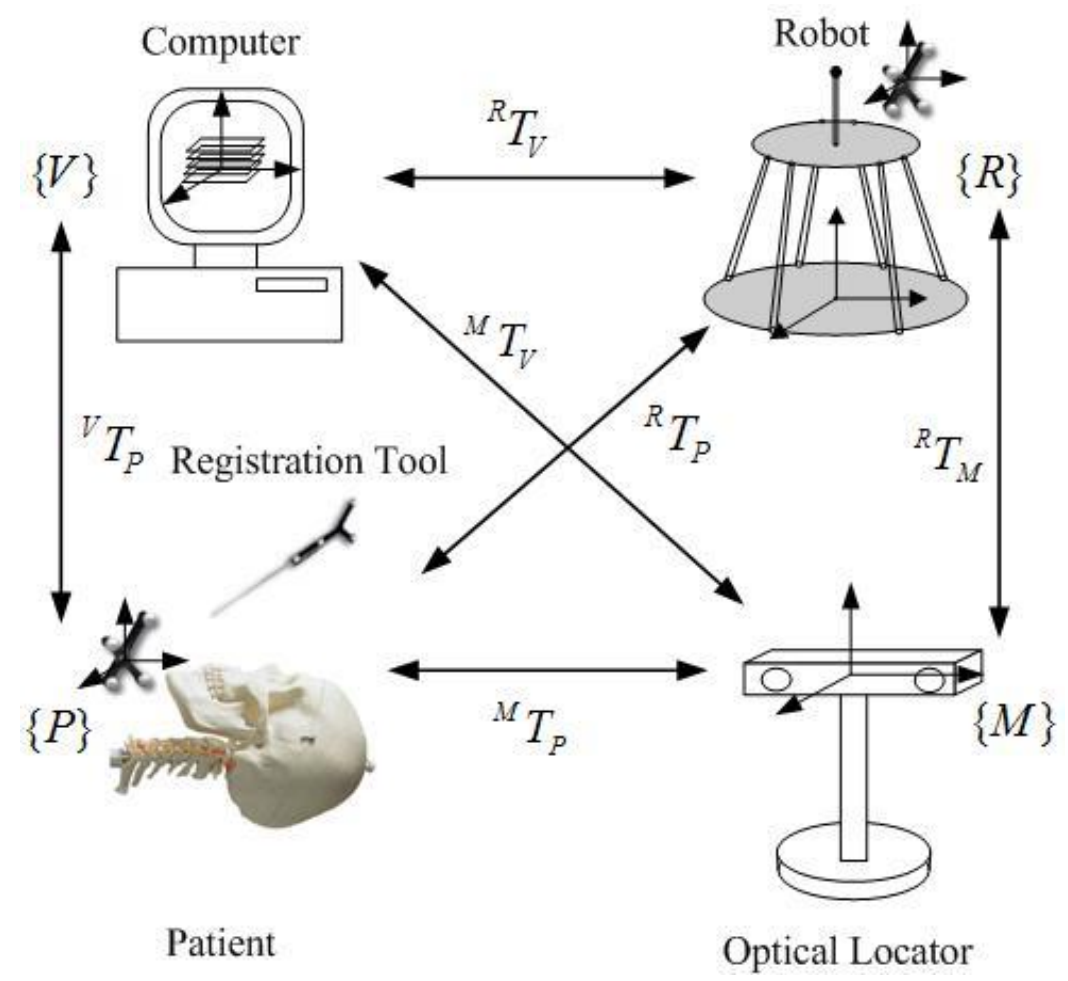

Fig. 2. Space transformations of robot-assisted surgical navigation for cervical vertebra bone grinding

\section{Key technologies of surgical navigation}

The key of robot-assisted surgical navigation for cervical vertebra bone grinding is coordinate transformations among different spaces and surgical instrument tracking and visualization. The position \& pose of bone-grinding robot end-effector planned in the computer image space must be transformed to the operation space of bone-grinding robot through space registration among "imagepatient-robot", so as to navigate and locate bone-grinding robot at the position \& pose pre-planned. Optical locator can be used to do real-time measurement of the position \& pose of bone-grinding robot end-effector relative to patient. The position \& pose information can be updated and displayed on the medical image in a real-time way in the form of virtual surgical instruments, thus to let surgeons see the position of surgical instruments relative to patients.

\subsection{ICP algorithm-based image-patient registration}

For difficulty in acquiring precise corresponding relation during registration of patient space 
and image space, Besl and McKay developed the ICP (Iterative Closest Point) algorithm based on Berthold algorithm [14]. ICP (Iterative Closest Point) algorithm has favorable effects on the registration of coordinate point sets of which definite corresponding relation is inconvenient or impossible to be learned. However, ICP algorithm is accompanied with characteristics of low calculation efficiency and easy to lead to local minimization problem; it cannot conduct correct registration especially when the rotational malposition of the two models is relatively large. This paper proposes a coordinate direction fitting-based ICP algorithm to acquire "image-patient" registration matrix to reduce rotational malposition between CT image space and patient space, solve local convergence of ICP algorithm and improve registration effects.

The method is to establish a patient coordinate system coinciding with coordinate system orientation of CT image by establishing patient coordinate system $\{P\}$ on a square. To facilitate the measurement, install a passive rigid body of optical tracking on the square with coordinate system $\{S\}$ as shown in Fig. 3a. The establishment method of direction fitting coordinates is described in detail in [15].After acquiring origin coordinates and three coordinate axis equations of coordinate system $P\left\{x_{p}, y_{p}, z_{p}\right\}$, homogeneous coordinate transformation matrix ${ }^{M} T_{P}$ of translation and rotation transformation under coordinate system $M\left\{x_{m}, y_{m}, z_{m}\right\}$ can be acquired as follows[15]:

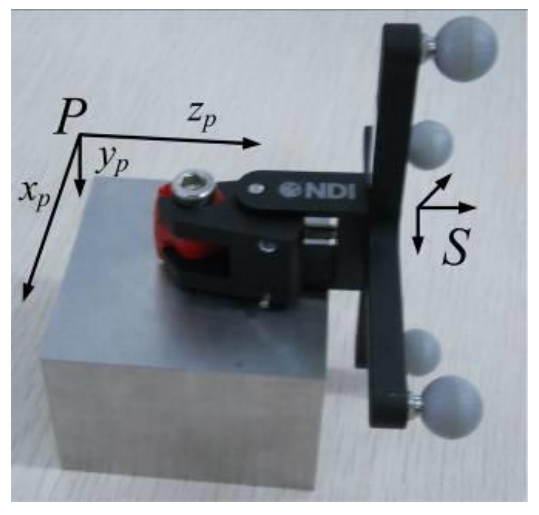

(a) A square with a passive rigid body

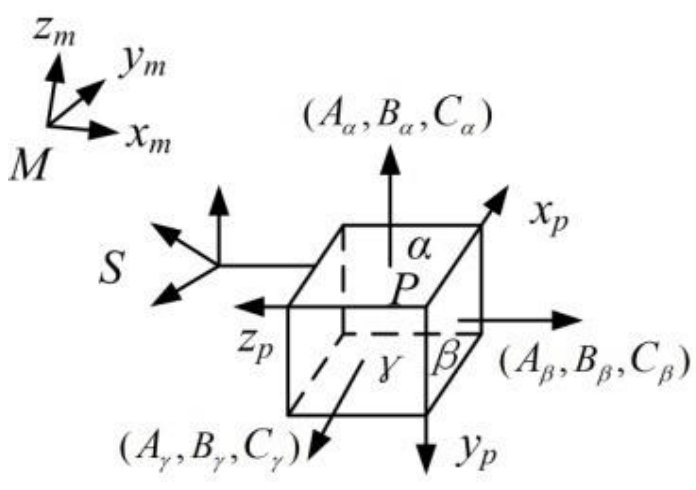

(b) Coordinate system definition

Fig. 3. Patient coordinate system definition 


$$
{ }^{M} T_{P}=\left[\begin{array}{cccc}
\frac{l_{x}}{L_{x}} & \frac{-A_{\alpha}}{L_{y}} & \frac{l_{z}}{L_{z}} & x_{m p} \\
\frac{m_{x}}{L_{x}} & \frac{-B_{\alpha}}{L_{y}} & \frac{m_{z}}{L_{z}} & y_{m p} \\
\frac{n_{x}}{L_{x}} & \frac{-C_{\alpha}}{L_{y}} & \frac{n_{z}}{L_{z}} & z_{m p} \\
0 & 0 & 0 & 1
\end{array}\right]
$$

Transformation matrix ${ }^{M} T_{p}$ from passive rigid body coordinate system $\{S\}$ to optical measuring coordinate system $\{M\}$ on the square can be acquired directly with optical locator, and then the transformation relation ${ }^{s} T_{p}$ from patient coordinate system to optical measuring coordinate system can be acquired as well. As shown in Fig. $4,{ }^{5} T_{p}$ is fixed, proper placing position of the square during surgery is fixed together with surgical bed according to actual conditions, making rotation direction of the patient coordinate system defined on the square almost coincide with that of CT coordinate system. In this way, coordinate point set of patient's cervical vertebral bone surface acquired with probe can be transformed into the patient coordinate system. Meanwhile, coordinate transformation ${ }^{v} T_{p}$ of CT image space and patient space can be implemented with ICP algorithm. The ${ }^{s} T_{p}$ calculation formula is as follows:

$$
{ }^{S} T_{P}=\left({ }^{M} T_{S}\right)^{-1}{ }^{M} T_{P}
$$

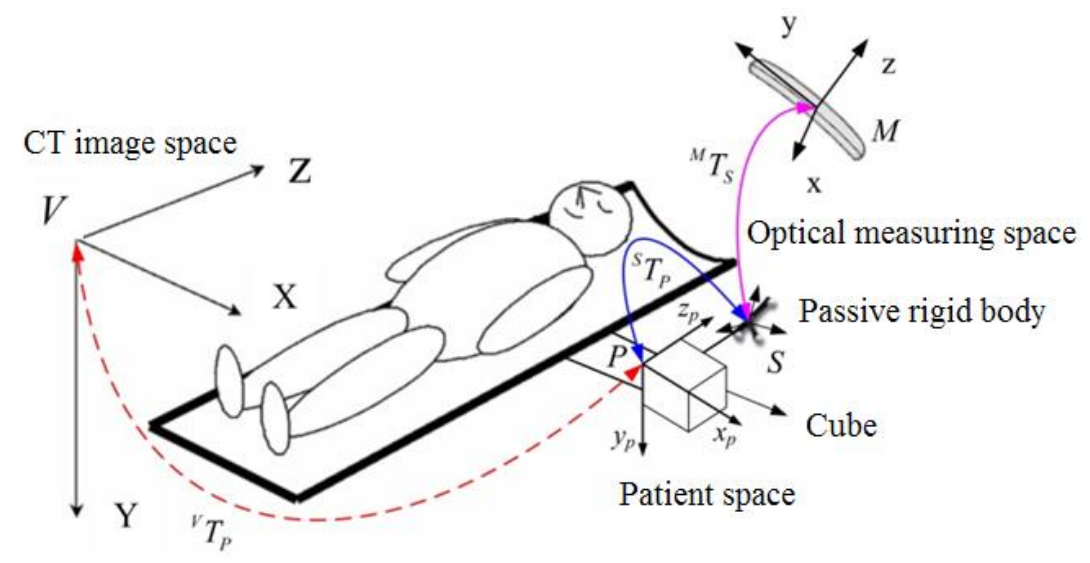

Fig. 4. Position relationships between CT image space and patient space 


\subsection{Planimetric method-based patient-robot space transformation}

Final goal of robot-assisted surgical navigation is to map information in image space into robot space. Therefore, transformation matrix between patient and robot is required. Take movable platform surface coordinate system $O^{\prime}\left\{x, y, z^{\prime}\right\}$ as measuring benchmark coordinate system, then acquire transformation matrix between the two with optical locator based on planimetric method.

\subsubsection{Principles of planimetric method}

As shown in Fig. 5, establish plane $\alpha$ on the upper surface of robot movable platform, while establishing planes $\beta$ and $\gamma$ on the installation surface of two adjacent spherical hinges. Planes $\beta$ and $\gamma$ are perpendicular to the connection between two hinge centers of movable platform and upper platform hinge distribution circle center respectively. The three planes $\alpha, \beta$ and $\gamma$ intersect at point $P$. Establish movable platform surface coordinate system $O^{\prime}\left\{\boldsymbol{x}, y^{\prime}, z^{\prime}\right\}$ as measuring benchmark coordinate system. Take movable platform surface coordinate system $O^{\prime}\{x, y, z\}$ as benchmark, translate coordinate system on $O^{\prime}\left\{x, y, z^{\prime}\right\}$ plane $\alpha$ to central plane of platform hinge along negative direction of $z$, axis by distance $d$, define the plane here as plane $\pi$ and coordinate system $O\left\{x_{p}, y_{p}, z_{p}\right\}$ as movable platform coordinate system of parallel robot. The transformation relation ${ }^{0} T_{0}$, between the two can be determined by $d$. When the robot is in zero position, position \& pose of movable platform surface coordinate system $O^{\prime}\{x, y, z\}$ is $\left(0,0, z_{p}, 0,0,0\right)$. Define robot base coordinate system $R\{\mathrm{x}, y, z\}$ with the position \& pose as benchmark. $z_{p}$ is determined with theoretical inverse kinematic model.

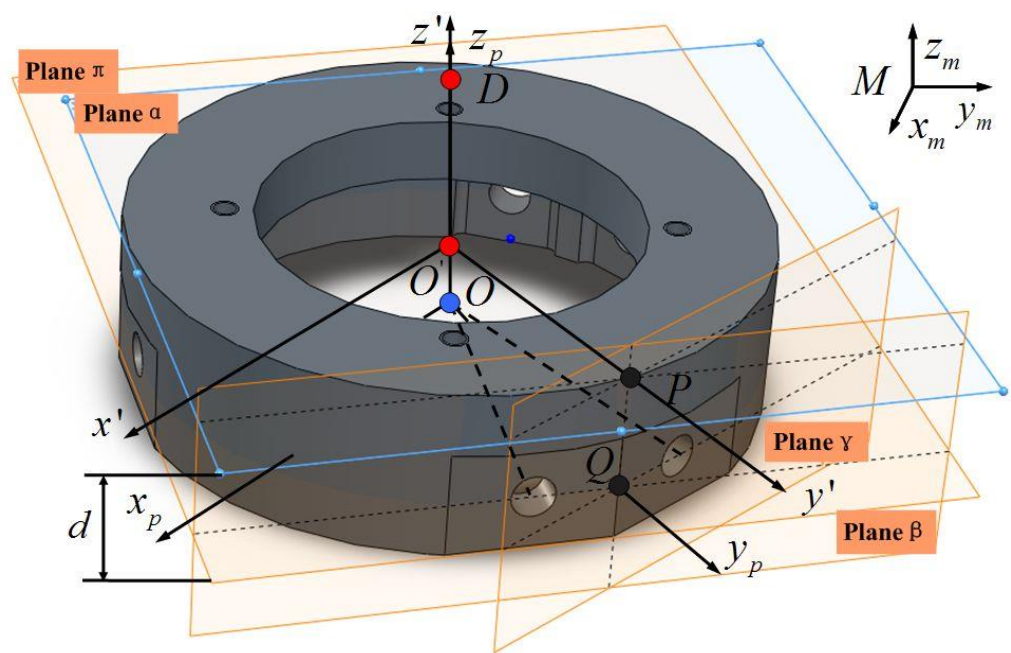

Fig. 5. Schematic diagram of planimetric method 
In Fig. 5, optical measuring coordinate system is defined as $M\left\{x_{m}, y_{m}, z_{m}\right\}$, where equations of planes $\alpha, \beta$ and $\gamma$ are defined as:

$$
\begin{aligned}
& A_{\alpha}\left(x-x_{\alpha}\right)+B_{\alpha}\left(y-y_{\alpha}\right)+C_{\alpha}\left(z-z_{\alpha}\right)=0 \\
& A_{\beta}\left(x-x_{\beta}\right)+B_{\beta}\left(y-y_{\beta}\right)+C_{\beta}\left(z-z_{\beta}\right)=0 \\
& A_{\gamma}\left(x-x_{\gamma}\right)+B_{\gamma}\left(y-y_{\gamma}\right)+C_{\gamma}\left(z-z_{\gamma}\right)=0
\end{aligned}
$$

In the optical measuring coordinate system $M\left\{x_{m}, y_{m}, z_{m}\right\}$, select no less than three points (noncollinear) on corresponding planes with optical positioning probe and determine equations of planes $\alpha, \beta$ and $\gamma$, then equation coefficients $A_{i}, B_{i}, C_{i}, x_{i}, y_{i}$ and $z_{i}(i=\alpha, \beta, \gamma)$ of three planes will also be acquired. Combine equations of planes $\alpha, \beta$ and $\gamma$ to work out coordinate $P\left\{x_{m p}, y_{m p}, z_{m p}\right\}$ of point $P$ in coordinate system $M\left\{x_{m}, y_{m}, z_{m}\right\}$, calculation formula is as follows:

$$
\left[\begin{array}{c}
x_{m P} \\
y_{m P} \\
z_{m P}
\end{array}\right]=R^{-1}\left[\begin{array}{l}
a_{\alpha} \\
b_{\beta} \\
c_{\gamma}
\end{array}\right]
$$

Where

$$
\begin{gathered}
R=\left[\begin{array}{ccc}
A_{\alpha} & B_{\alpha} & C_{\alpha} \\
A_{\beta} & B_{\beta} & C_{\beta} \\
A_{\gamma} & B_{\gamma} & C_{\gamma}
\end{array}\right] \quad, \quad a_{\alpha}=A_{\alpha} x_{\alpha}+B_{\alpha} y_{\alpha}+C_{\alpha} z_{\alpha} \quad, \quad b_{\beta}=A_{\beta} x_{\beta}+B_{\beta} y_{\beta}+C_{\beta} z_{\beta}, \\
c_{\gamma}=A_{\gamma} x_{\gamma}+B_{\gamma} y_{\gamma}+C_{\gamma} z_{\gamma} .
\end{gathered}
$$

In addition, coordinate value $D\left\{x_{m D}, y_{m D}, z_{m D}\right\}$ of grinding cutter-end point can be measured with a probe. Normal vector $\left\{A_{\alpha}, B_{\alpha}, C_{\alpha}\right\}$ of plane $\alpha$ can be acquired according to plane $\alpha$ equation, then equation of straight line $O^{\prime} D$ where $z^{\prime}$ axis of coordinate system $O^{\prime}\left\{x, y, z^{\prime}\right\}$ can be obtained. Coordinate value $O^{\prime}\left\{x_{m} 0^{\prime}, y_{m} 0^{\prime}, z_{m} 0^{\prime}\right\}$ of coordinate system $O^{\prime}\left\{x, y, z^{\prime}\right\}$ can be acquired by combining 
$z^{\prime}$ axis equation and plane $\alpha$ equation of the coordinate system. Measure coordinates $P\left\{x_{m p}, y_{m p}, z_{m p}\right\}$ and $O^{\prime}\left\{x_{m 0^{\prime}}, y_{m 0^{\prime}}, z_{m 0^{\prime}}\right\}$ respectively which have been worked out under coordinate system $M\left\{x_{m}, y_{m}\right.$, $\left.z_{m}\right\}$ with optical locator, then equation of straight line $O^{\prime} P$ where $y$, axis of coordinate system $O^{\prime}\{x$, $\left.y^{\prime}, z^{\prime}\right\}$ lies in can be acquired. Assume normal vectors of $x, y$, and $z$, axis as $l, m$ and $n$ respectively, then equation of $y$, axis of coordinate system $O^{\prime}\left\{x, y, z^{\prime}\right\}$ is also obtained. Three equations are shown as follows:

$z^{\prime}$ axis equation:

$\frac{\left(x-x_{m D}\right)}{A_{\alpha}}=\frac{\left(y-y_{m D}\right)}{B_{\alpha}}=\frac{\left(z-z_{m D}\right)}{C_{\alpha}}$

$y^{\prime}$ axis equation:

$\frac{\left(x-x_{m O^{\prime}}\right)}{\left(x_{m P}-x_{m O^{\prime}}\right)}=\frac{\left(y-y_{m O^{\prime}}\right)}{\left(y_{m P}-y_{m O^{\prime}}\right)}=\frac{\left(z-z_{m O^{\prime}}\right)}{\left(z_{m P}-z_{m O^{\prime}}\right)}$

$x^{\prime}$ axis equation:

$$
\frac{\left(x-x_{m O^{\prime}}\right)}{l_{x}}=\frac{\left(y-y_{m O^{\prime}}\right)}{l_{y}}=\frac{\left(z-z_{m O^{\prime}}\right)}{l_{z}}
$$

Where

$$
\begin{aligned}
& l=\left\{l_{x}, l_{y}, l_{z}\right\}, m=\left\{m_{x}, m_{y}, m_{z}\right\}=\left\{x_{m P}-x_{m O^{\prime}}, y_{m P}-y_{m O^{\prime}}, z_{m P}-z_{m O^{\prime}}\right\}, n=\left\{n_{x}, n_{y}, n_{z}\right\}=\left\{A_{\alpha}, B_{\alpha}, C_{\alpha}\right\}, \\
& l=m \times n=\left|\begin{array}{ccc}
i & j & k \\
m_{x} & m_{y} & m_{z} \\
l_{x} & l_{y} & l_{z}
\end{array}\right|=\left|\begin{array}{ccc}
i & j & k \\
x_{m P}-x_{m O^{\prime}} & y_{m P}-y_{m O^{\prime}} & z_{m P}-z_{m O^{\prime}} \\
A_{\alpha} & B_{\alpha} & C_{\alpha}
\end{array}\right|
\end{aligned}
$$

After acquiring zero point and axis equation of coordinate system $O^{\prime}\left\{x, y^{\prime}, z^{\prime}\right\}$, transformation 
matrix ${ }^{M} T_{0}{ }^{\prime}$ of translation and rotation homogeneous coordinates under coordinate system $M\left\{x_{m}, y_{m}\right.$, $\left.z_{m}\right\}$ at zero position can be worked out as follows:

$$
{ }^{M} T_{O^{\prime} 0}=\left[\begin{array}{cccc}
\frac{l_{x}}{V_{x}} & \frac{m_{x}}{V_{y}} & \frac{n_{x}}{V_{z}} & x_{m O^{\prime}} \\
\frac{l_{y}}{V_{x}} & \frac{m_{y}}{V_{y}} & \frac{n_{y}}{V_{z}} & y_{m O^{\prime}} \\
\frac{l_{z}}{V_{x}} & \frac{m_{z}}{V_{y}} & \frac{n_{z}}{V_{z}} & z_{m O^{\prime}} \\
0 & 0 & 0 & 1
\end{array}\right]
$$

Where

$$
V_{x}=\sqrt{l_{x}^{2}+l_{y}^{2}+l_{z}^{2}}, V_{y}=\sqrt{m_{x}^{2}+m_{y}^{2}+m_{z}^{2}}, V_{z}=\sqrt{n_{x}^{2}+n_{y}^{2}+n_{z}^{2}}
$$

\subsubsection{Patients-robots space transformation}

As shown in Fig.6, to acquire the transformation matrix ${ }^{R} T_{M}$ between optical measuring coordinate system $M\left\{x_{m}, y_{m}, z_{m}\right\}$ and parallel robot base coordinate system $R\{x, y, z\}$ more conveniently, and to install passive rigid body of optical tracking, a tracking tool coordinate system $T\left\{x_{t}, y_{t}, z_{t}\right\}$ was defined. The transformation matrix ${ }^{M} T_{T}$ between optical measuring coordinate system $M\left\{x_{m}, y_{m}, z_{m}\right\}$ and tracking tool coordinate system $T\left\{x_{t}, y_{t}, z_{t}\right\}$ can be implemented and acquired. Only need to find the transformation matrix ${ }^{T} T_{0}$, between movable platform planes coordinate system $O^{\prime}\left\{x, y^{\prime}, z^{\prime}\right\}$ and tracking tool coordinate system $T\left\{x_{t}, y_{t}, z_{t}\right\}$ when using it. When the parallel robot is in zero position, ${ }^{M} T_{T O}$ can be acquired through the theory of planimetric method, thus matrix ${ }^{T} T_{0}$, can be acquired through the following formula:

$$
{ }^{T} T_{O^{\prime}}=\left({ }^{M} T_{T 0}\right)^{-1}{ }^{M} T_{O^{\prime} 0}
$$




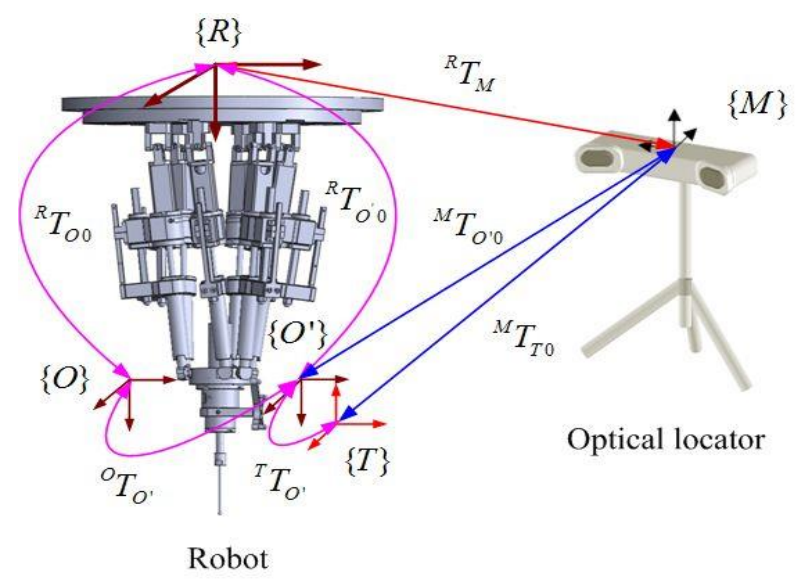

Fig. 6. Mapping transformations from optical measuring space to robot base coordinate system

The transformation matrix ${ }^{T} T_{0}$, between movable platform planes coordinate system $O^{\prime}\{x, y$, $\left.z^{\prime}\right\}$ and tracking tool coordinate system $T\left\{x_{t}, y_{t}, z_{t}\right\}$ is the fixed matrix of space transformation of robot. Using this matrix, the space registration transformation between coordinate systems of robot space can be implemented. When parallel robot is at zero position, the transformation relation ${ }^{R} T_{00}$ between movable platform space and fixed platform space in theoretical model is given, and the transformation matrix ${ }^{R} T_{00}$ from robot movable platform plane coordinate system $O^{\prime}\left\{x, y^{\prime}, z^{\prime}\right\}$ to movable platform coordinate system $O\left\{x_{p}, y_{p}, z_{p}\right\}$ is also given, in this way, when parallel robot is at zero position, the transformation matrix ${ }^{R} T_{0}{ }_{0}$ from movable platform planes coordinate system $O^{\prime}\{x$, $\left.y, z^{\prime}\right\}$ to robot base coordinate system $R(x, y, z)$ can be acquired. When the space transformation between optical measuring coordinate system $M\left\{x_{m}, y_{m}, z_{m}\right\}$ and parallel robot base coordinate system $R(x, y, z)$ is needed, the transformation matrix ${ }^{R} T_{M}$ between optical measuring coordinate system $M\left\{x_{m}, y_{m}, z_{m}\right\}$ and parallel robot base coordinate system $R(x, y, z)$ can be acquired conveniently when the movable platform is at zero position through the transformation matrix ${ }^{T} T_{0}$, between movable platform planes coordinate system $O^{\prime}\left\{x, y, z^{\prime}\right\}$ and tracking tool coordinate system $T\left\{x_{i}, y_{i}, z_{i}\right\}$. Then, the space transformation matrix ${ }^{R} T_{p}$ from patient coordinate system $\{P\}$ to parallel robot base coordinate system $\{R\}$ is

$$
{ }^{R} T_{P}={ }^{R} T_{M}{ }^{M} T_{P}={ }^{R} T_{O^{\prime} 0}\left({ }^{T} T_{O^{\prime}}\right)^{-1}\left({ }^{M} T_{T 0}\right)^{-1}{ }^{M} T_{P}
$$

Where 


$$
\begin{aligned}
& { }^{R} T_{M}={ }^{R} T_{O^{\prime} 0}\left({ }^{T} T_{O^{\prime}}\right)^{-1}\left({ }^{M} T_{T 0}\right)^{-1} \\
& { }^{R} T_{O^{\prime} 0}={ }^{R} T_{O 0}{ }^{O} T_{O^{\prime}}
\end{aligned}
$$

Grinding tool-end coordinate system is $D\left\{x_{d}, y_{d}, z_{d}\right\}$, which is acquired after movable platform coordinate system $O^{\prime}\{x, y, z\}$ moves $z$, along its forward direction $l$, and it has the same position \& pose as movable platform planes coordinate system $O^{\prime}\{x, y, z\}$. Constant $l$ is set according to the length of cutter, different grinding tools are installed, and Constant $l$ has different numerical values. Therefore, based on the transformation matrix between grinding tool-end coordinate system $D\left\{x_{d}, y_{d}\right.$, $\left.z_{d}\right\}$ and movable platform planes coordinate system $O^{\prime}\left\{x, y, z^{\prime}\right\}$, the transformation matrix ${ }^{T} T_{D}$ of grinding tool-end coordinate system $D\left\{x_{d}, y_{d}, z_{d}\right\}$ related to tracking tool coordinate system $T\left\{x_{t}, y_{t}\right.$, $\left.z_{t}\right\}$ can be acquired. This matrix ${ }^{T} T_{D}$ is fixed and related to grinding tool, which can be acquired by using the following formula:

$$
{ }^{T} T_{D}={ }^{T} T_{O^{\prime}}{ }^{O^{\prime}} T_{D}
$$

Where

$$
{ }^{o} T_{D}=\left[\begin{array}{llll}
1 & 0 & 0 & 0 \\
0 & 1 & 0 & 0 \\
0 & 0 & 1 & l \\
0 & 0 & 0 & 1
\end{array}\right]
$$

When the movable platform is in any position \& pose, the mapping matrix ${ }^{M} T_{D}$ from grinding tool-end coordinate system $\{D\}$ to optical measuring coordinate system $\{D\}$ can be acquired through passive rigid body of real-time tracking. At last, the transformation matrix ${ }^{T} T_{D}$ from grinding toolcoordinate system $\{D\}$ to patient coordinate system $\{P\}$ can be acquired through transformation matrix ${ }^{R} T_{D}$ : 


$$
{ }^{P} T_{D}=\left({ }^{M} T_{P}\right)^{-1}{ }^{M} T_{D}=\left({ }^{M} T_{P}\right){ }^{-1 T} T_{D}\left({ }^{M} T_{T}\right)^{-1}
$$

Where

$$
{ }^{M} T_{D}={ }^{T} T_{D}\left({ }^{M} T_{T}\right)^{-1}
$$

\subsection{Real-time tracking and visualization}

As is shown in Fig. 7, transformation matrix ${ }^{V} T_{p}$ between spine model coordinate system $\{V\}$ in image space and patient spine coordinate system $\{P\}$ can be implemented through space registration. Among them, the transformation matrix ${ }^{M} T_{p}$ between optical locator $\{M\}$ and patient spine $\{P\}$ as well as the transformation matrix ${ }^{M} T_{T}$ between optical locator $\{M\}$ and tool coordinate system $\{T\}$ can be acquired through the measurement of optical locator. The coordinate transformation ${ }^{T} T_{D}$ between grinding tool-end coordinate system $\{D\}$ and tool coordinate system $\{T\}$ can be obtained through Equ.21. Optical locator can real-time track tool coordinate system $\{T\}$ by a passive rigid body, thus the transformation matrix ${ }^{V} T_{D}$ between grinding tool-end $D$ and coordinate system $\{V\}$ of 3D model in image space can be acquired at any moment:

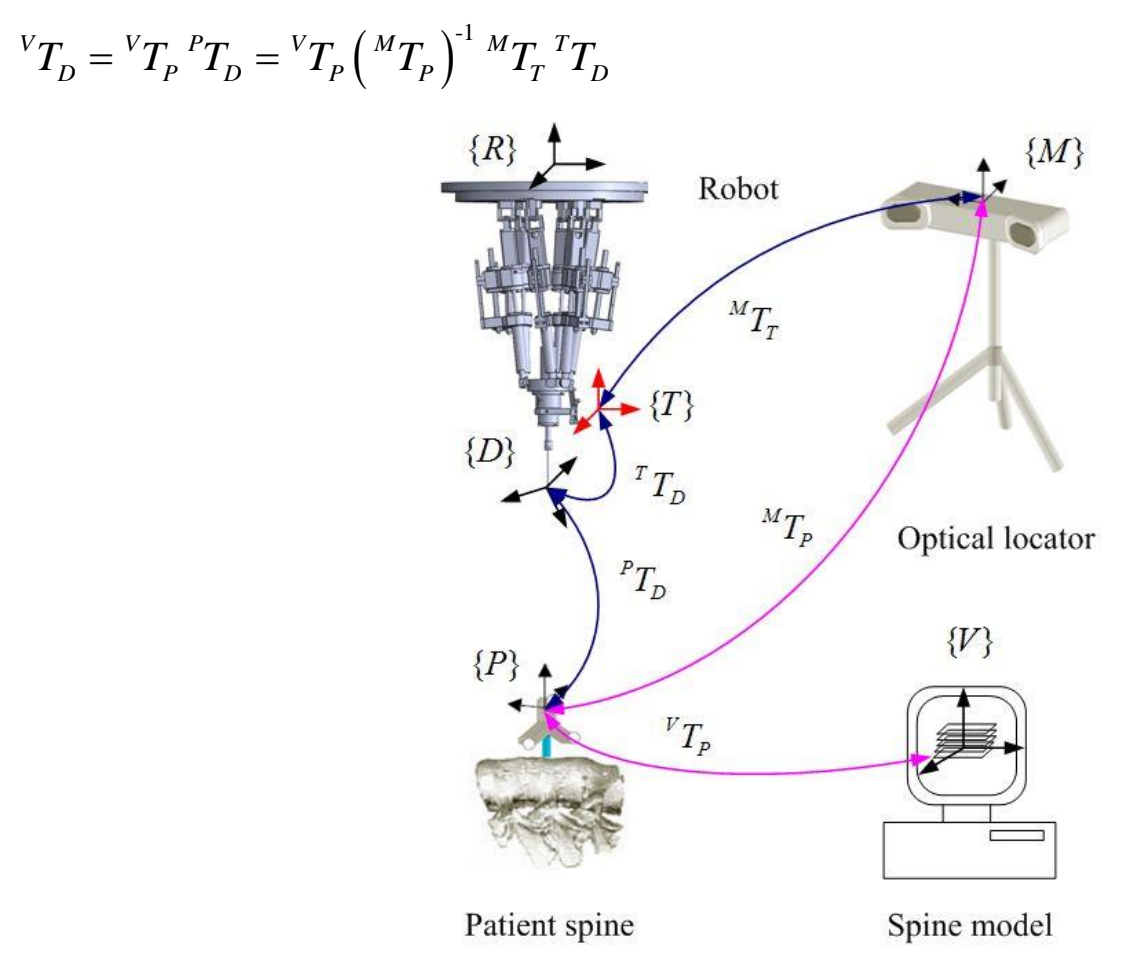

Fig. 7. Matrix transformations for robot end-effectors' real-time tracking and display 
To explain the internal mechanism of real-time tracking of bone-grinding robot end-effector, the tracking matrix ${ }^{V} T_{D}$ of bone-grinding robot end-effector relative to image space is assumed as:

$$
{ }^{V} T_{D}=\left[\begin{array}{cc}
R_{3 \times 3} & T \\
0 & 1
\end{array}\right]
$$

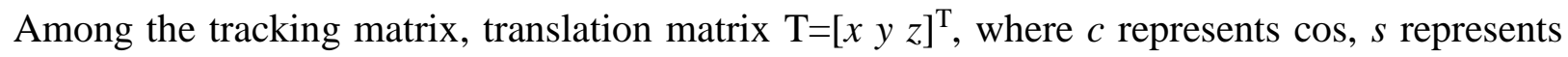
sin, and rotation transformation matrix $R_{3 \times 3}$ is:

$$
R_{3 \times 3}=R(x, \alpha) R(y, \beta) R(z, \gamma)=\left[\begin{array}{ccc}
\mathrm{c} \beta \mathrm{c} \gamma & -\mathrm{c} \beta \mathrm{s} \gamma & \mathrm{s} \beta \\
\mathrm{s} \alpha \mathrm{s} \beta \mathrm{c} \gamma+\mathrm{c} \alpha \mathrm{s} \gamma & \mathrm{c} \alpha \mathrm{c} \gamma-\mathrm{s} \alpha \mathrm{s} \beta \mathrm{s} \gamma & -\mathrm{s} \alpha \mathrm{c} \beta \\
\mathrm{s} \alpha \mathrm{s} \gamma-\mathrm{c} \alpha \mathrm{s} \beta \mathrm{c} \gamma & \mathrm{c} \alpha \mathrm{s} \beta \mathrm{s} \gamma+\mathrm{s} \alpha \mathrm{c} \gamma & \mathrm{c} \alpha \mathrm{c} \beta
\end{array}\right]
$$

\section{Results and discussion}

By measurement of actual position \& pose of robot end-effector and comparison with planning paths, bone-grinding robot navigation function and precision can be verified. And navigating and positioning precision of robot-assisted spine surgery can be analyzed by performing positioning and tracking experiments.

\subsection{Verification experiment of surgical navigation}

Experiment system is set up as shown in Fig. 8, which includes a navigation computer, a bone grinding robot and an optical positioning system. The passive optical positioning system Polaris Spectra made in Canada NDI has high 3D space positioning accuracy and wide vision tracking. The robot position and pose measuring can be completed by using the optical probe and passive rigid body. 

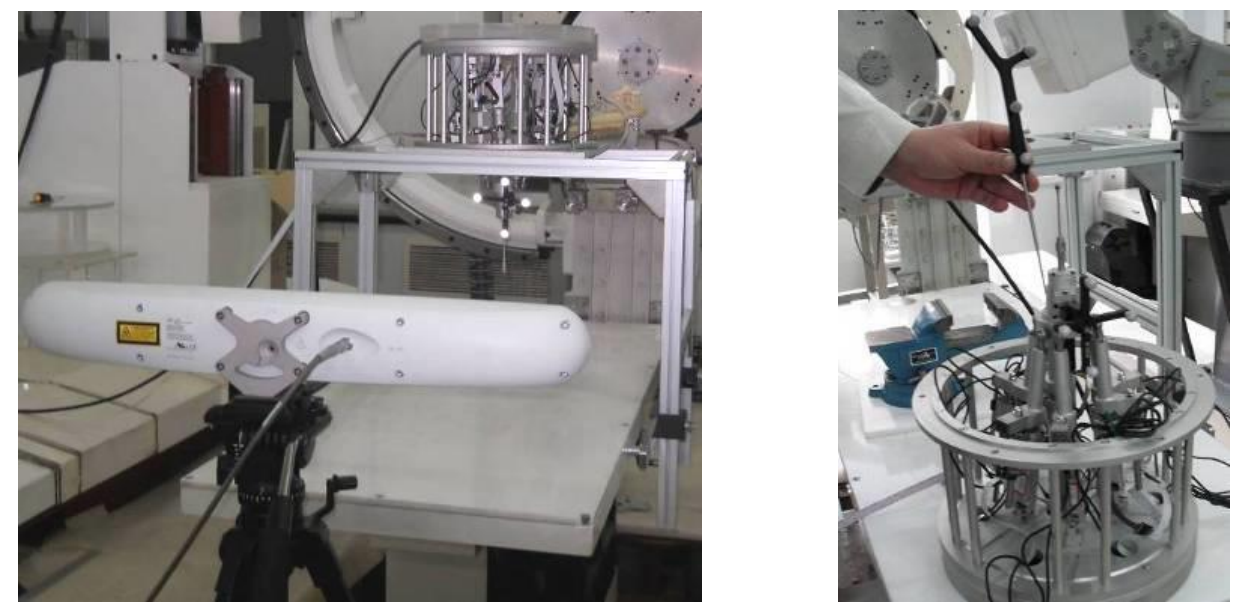

(a) Experiment system

(b) Position and pose measuring

Fig. 8. Experimental platform for surgical navigation

Firstly, CT scanning of experimental model is conducted, and then reconstructs the 3D structure of this model. Use three points planning to plan the cutter position \& pose before surgery. The planned three planning paths are shown in Fig. 9. Use three points planning to calculate the transformation matrix of cutter planning coordinate system $K\left\{x_{k}, y_{k}, z_{k}\right\}$ relative to image space $\{V\}$. Secondly, use the registration method between "image-patient" based on ICP algorithm to acquire the transformation matrix ${ }^{P} T_{V}$ of image space and patient space; the acquiring methods of registration points as shown in Fig. 10 and Fig.11. Then, use the "patient-robot" space transformation technology based on planimetric method to acquire the transformation matrix ${ }^{R} T_{P}$ of patient space and robot space. Using the above matrix, surgical navigation matrix ${ }^{R} T_{K}$ can be acquired, and the calculation formula of ${ }^{R} T_{K}$ is Equ.24. Finally, use matrix ${ }^{R} T_{K}$ of robot-assisted surgical navigation to guide the surgery. The image guiding effect is as shown in Fig. 12.

$$
{ }^{R} T_{K}={ }^{R} T_{V}{ }^{V} T_{K}={ }^{R} T_{P}{ }^{P} T_{V}{ }^{V} T_{K}
$$




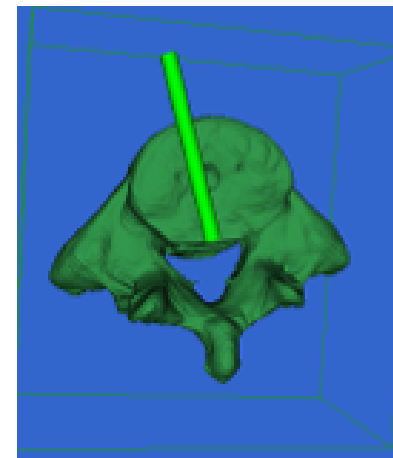

(a) Left path

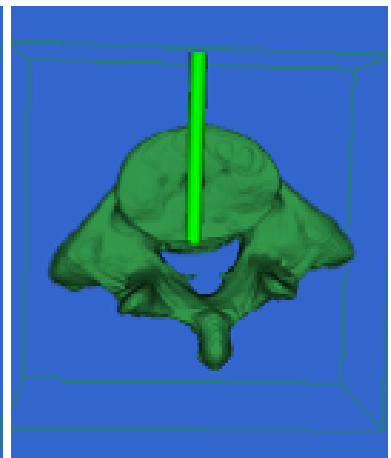

(b) Median path

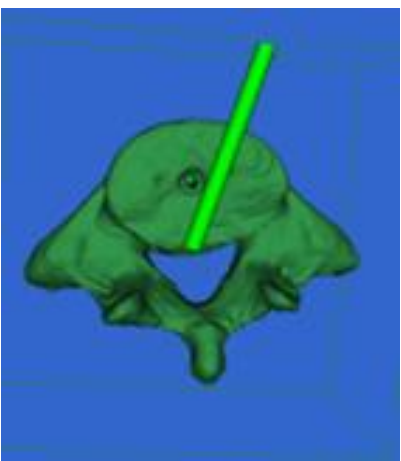

(c) Right path

Fig. 9. Three planning paths
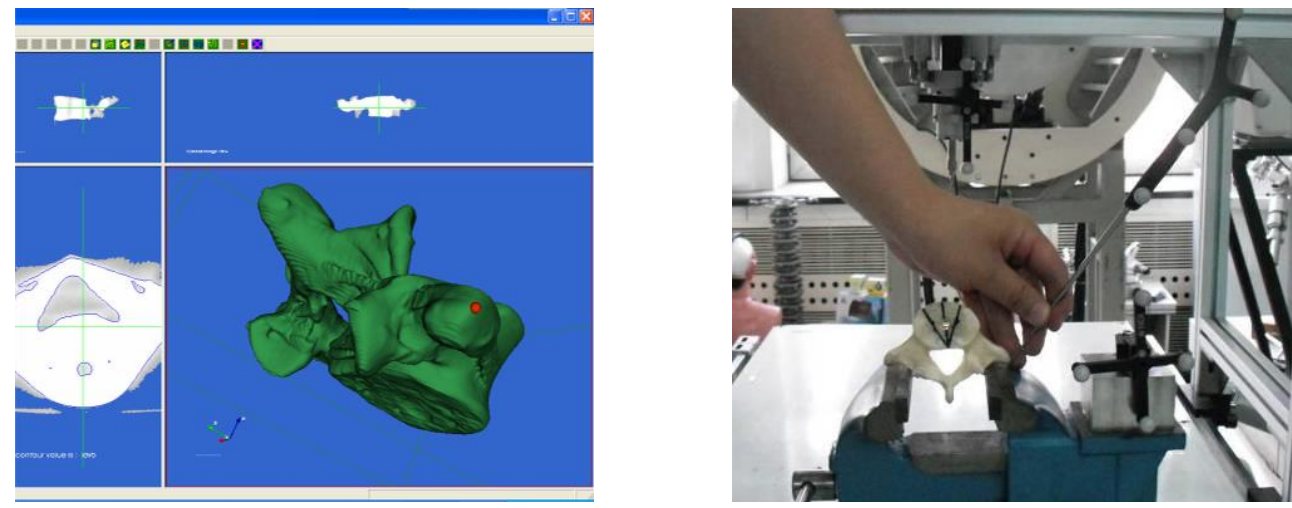

Fig. 10. Registration points in image space Fig. 11. Registration points in patient space

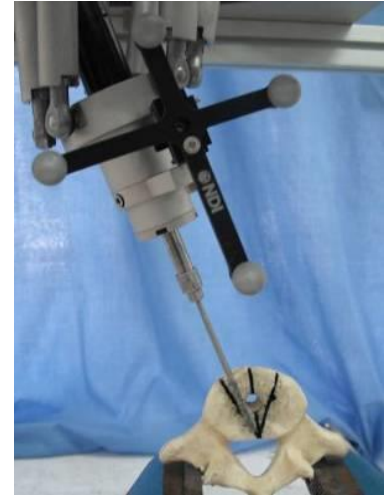

(a) Left path guiding

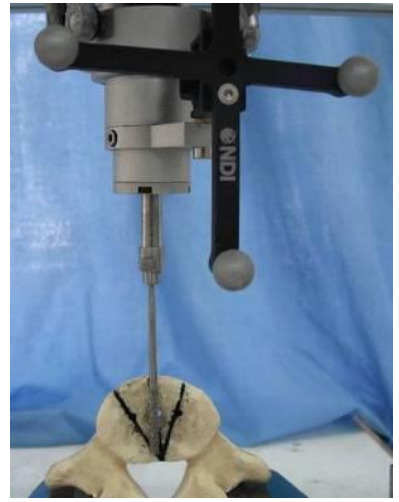

(b) Median path guiding

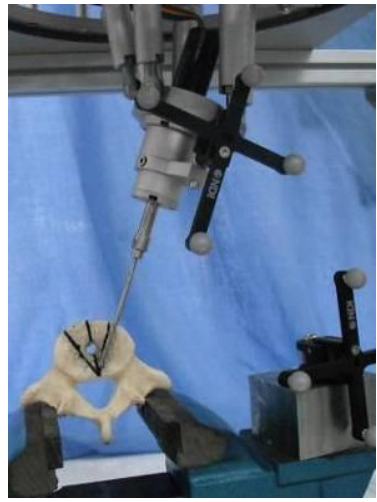

(c) Right path guiding

Fig. 12. Navigation effect guided by surgical paths

\subsection{Analyzing of navigation and positioning precision}

Use the planned right surgical path to test the navigation and positioning precision. Firstly, 
transform the left surgical path to robot space; then, use the dynamitic model of robot to control the robot to do position \& pose adjustment. In ideal condition, the position \& pose of robot end-cutter shall be in accordance with that of planning path; but in practical condition, there is deviation between planning path and practical path. Navigating and positioning precision is analyzed through calculation. In the experiment, 10 registration experiments are conducted through the registration method between "image-patient" based on ICP algorithm so as to acquire the transformation matrix ${ }^{R} T_{V}$ of image space and patient space; then, use the "patient-robot" space transformation technology based on planimetric method to acquire the transformation matrix ${ }^{R} T_{P}$ of patient space and robot space, thus to acquire matrix ${ }^{R} T_{K}$ of the 10 surgeries. The end-point of right path is used as positioning point. Use this navigation matrix to guide surgery; use optical locator to measure the robot end-position \& pose for 10 times respectively; compare the measuring results and position \& pose of practical path so as to calculate the positioning errors.

The calculated result is shown in Fig. 13, error distribution figures of the 10 experiments are drawn according to position errors and poses errors. From the Fig. 13a, it can be observed that the positioning errors are within 3.0-4.0 mm with a maximum position error of $4.0 \mathrm{~mm}$; and from Fig. $13 \mathrm{~b}$, it can be observed that the pose errors are within $2.7-3.4^{\circ}$ with a maximum pose error of $3.4^{\circ}$. To apply this system in clinic, it is necessary to acquire higher precision compared with manual replacement surgery of cervical intervertebral disc, and to further improve the precision of robotassisted system of replacement surgery of cervical intervertebral disc.

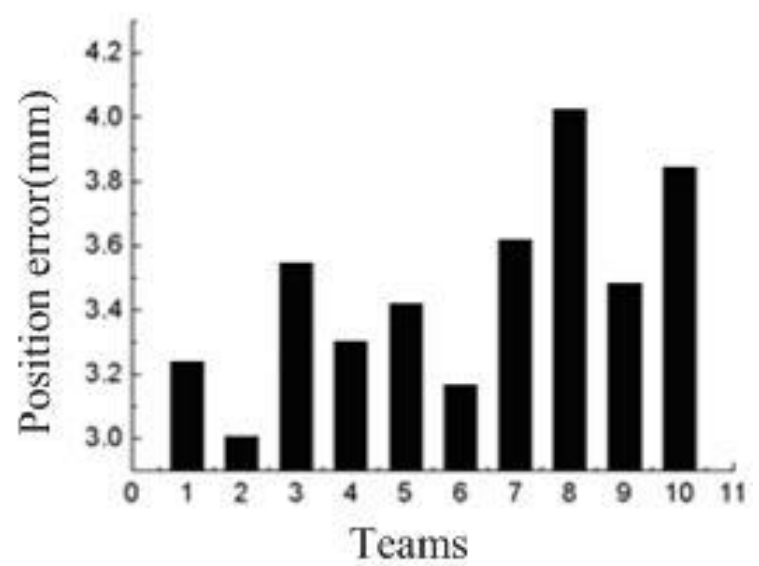

(a) Position errors

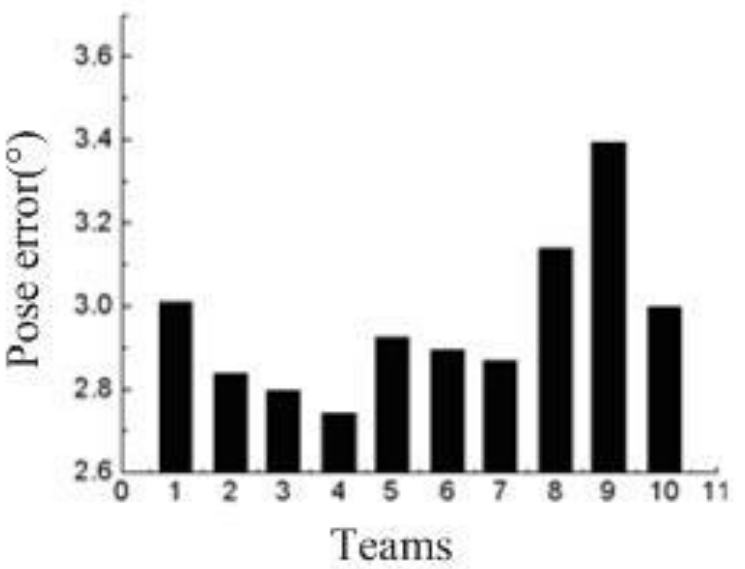

(b) Pose errors

Fig. 13. Positioning errors for robot-assisted navigation 
Orthopaedic surgery robot has a more obvious advantage than doctors in performing surgery planning, but which requires surgical robot systems to combine preoperative surgery planning with intraoperative actual situation by considering modeling problems of patients and relative positioning and navigating problems of surgical instruments [16]. Because personalized model of patients arises from preoperative and intraoperative acquisition of medical image data, the nonlinear calibration errors as a result of the soft tissue deformation will cause the deviation of robotic surgery eventually. The relative positioning precision between surgical instruments and patients decides the system surgical planning precision. At the same time, high precision dynamic tracking can improve the operation efficiency and safety. Positioning accuracy of surgical instruments caused by the influence of preoperative image, registration error, surgical instruments, sensors assembling error and surgical instruments vibration, and so on. From the errors analysis, it can be observed that major errors come from space transformation of "image-patient-robot", which includes the errors of intraoperative registration and the errors of parallel robot itself. Therefore, to acquire higher precision of surgical positioning, the following two aspects are emphasized:

(1) Further improve surgical model and intraoperative registration precision between "imagepatient", and emphasize on improving precision and speed of registration in follow-up researches. To improve the surgical navigation precision, 2D/3D registration of image can be implemented [17, 18]. This method uses intraoperative real-time X-ray image and preoperative CT image to implement registration, which is of high precision and good robustness. It is also easy to implement the automation, and will not cause any extra damage to patients. It meets the demands of minimally invasive surgery, and has a great prospect;

(2) To improve the precision of parallel robots, the precision of robots themselves will be improved from the aspects of processing, assembly and standardization. Generally, there are the following two methods: the first is to implement processing methods of high precision and assembly technology of high precision to manufacture and assembly robots; the second is to apply measuring instrument of high precision to make standardization and error compensation of robots by calibration method [19-21].

\section{Conclusions}


The accuracy, flexibility, and stability of surgical robot have been recognized by the medical community and the patients. Surgery robot has become an important method to achieve orthopedic surgery with the minimal invasiveness, digitization and intelligence. Orthopedic surgery has its own characteristics on surgical objective, surgical instruments, operating mode, and so on. Especially, the orthopedic surgical robot highly depends on the intraoperative navigation devices, which determines the technical specificity of orthopedic surgical robot and has broad clinical application. This paper introduces robot technology and image navigation technology into for cervical vertebra bone grinding in spine surgery, and builds robot-assisted surgical navigation system for cervical vertebra bone grinding in spine surgery. Aiming at the space registration of "image-patient-robot", ICP algorithm that is fitted based on the direction of coordinate system is used to acquire the registration matrix between "image-patient". Planimetric method is applied to implement space transformation between patient and robot. To verify the functionality and affectivity of robot-assisted surgical navigation, analysis of precision and errors of navigation and positioning is conducted by performing positioning and tracking experements. The experiment results show that the established active surgical navigation system can achieve positioning and tracking of bone-grinding robot feasibly and rationally. But, improvement means can be applied to further improve the precision of the system.

\section{ACKNOWLEDGEMENT}

The authors would like to express appreciation to financial supports from China Postdoctoral Science Foundation Funded Project (2016M602164), Qingdao Postdoctoral Researchers Applied Research Project (2016119), Outstanding Young Scientist in Shandong Province (2014SBS1415), Scientific Research Foundation of the SUST University (2014RCJJ023).

\section{REFERENCES}

1. Tsai TC, Hsu YL, Development of a parallel surgical robot with automatic bone drilling carriage for stereotactic neurosurgery, 2007, Biomed Eng Appl Basis Commun, vol.19, pp.26977.

2. Kuang SL, Leung KS, Wang TM, Chui LH, Liu WY, Wang Y, A novel passive/active hybrid robot for orthopaedic trauma surgery,2012, Int J Med Robotics Comput Assist Surg, vol.8,pp.458-67. 
3. Qiao F, Li D, Jin Z, et al, Application of 3D printed customized external fixator in fracture reduction, 2015, Injury-international Journal of the Care of the Injured, vol.46, no.6, pp.11501155.

4. Paula Gomes, Surgical robotics: Reviewing the past, analyzing the present, imagining the future, 2011, Robotics and Computer-Integrated Manufacturing, vol.27, no.2, pp.261-266.

5. Bargar WL, Robots in orthopaedic surgery: past, present, and future, 2007, Clinical Orthopaedics and Related Research, vol.463, no.463, pp.31-36.

6.Taylor RH, Mittelstadt BD , Paul HA, Hanson W, Kazanzides P, Zuhars JF , Williamson B, Musits BL, Glassman E, Bargar WL, An Image- Directed Robotic System for Precise Orthopaedic Surgery,1994, IEEE Trans. Robot and Automation,vol.10,pp. 261-275.

7. Cobb J, Henckel J, Gomes P, et al, Hands-on robotic unicompartmental knee replacement: A prospective, randomised controlled study of the acrobot system, 2006, J Bone Joint Surg Br, vol.88, pp.188-197.

8. Hagag B, Abovitz R, Kang H, et al, RIO: Robotic-arm interactive orthopedic system MAKOplasty: User interactive haptic orthopedic robotics, 2011, Surgical Robotics. New York: Springer, pp.219-246.

9.Pechlivanis I, Kiriyanthan G, Engelhardt M, Scholz M, Lücke S, Harders A, Schmieder K, Percutaneous placement of pedicle screws in the lumbar spine using a bone mounted miniature robotic system: first experiences and accuracy of screw placement, 2009, Spine, vol.34, no.4, pp.392-398.

10. Larsson N, Molin L, Rapid Prototyping of User Interfaces in Robot Surgery - Wizard of Oz in Participatory Design, 2006, Advances in Information Systems Development, Springer US, pp.361-371.

11.Chung GB, Kim S, Lee SG, Yi B, Kim W, Oh SM, Kim YS, So BR, Park JI, Oh SH, An Image-Guided Robotic Surgery System for Spinal Fusion, 2006, International Journal of Control Automation and Systems, vol.4, no.1,pp.30-41.

12. Hu L, Luan S, Wang M Y, et al, A biplanar robot navigation system for the distal locking of intramedullary nails, 2010, Int J Med Robot Comp, vol.6,pp.61-65.

13.Sun Lining, Zhang Jian, Du Zhijiang, A Robot-assisted System of Bone Surgery Based on Image Navigation,2006, College Journal of Harbin Engineering University, vol.27, no.2, pp. 
285-289.

14.Besl P J, McKay ND, A method for registration of 3D shapes,1992, IEEE Transactions on pattern analysis and machine intelligence, vol.14, pp.239-256.

15.Heqiang Tian, Peng Yang, Chunjian Su and Zhiqiang Dong, ICP Registration Technology based on the Coordinate System Direction Fit, 2015, International Journal of Security and Its Applications vol.9, no.12, pp.47-56.

16.Song G L, Han J D, Zhao Y W, Review of orthopedic surgical robot and navigation technology (in Chinese), 2013, Chin Sci Bull (Chin Ver), vol. 58(Supp.II), pp.8-19.

17.Valenti M, Ferrigno G, Martina D, et al, Gaussian mixture models based 2D-3D registration of bone shapes for orthopedic surgery planning, 2016, Medical \& Biological Engineering, pp.11-14.

18. Ketcha M D, De S T, Uneri A, et al, Automatic Masking for Robust 3D-2D Image Registration in Image-Guided Spine Surgery, 2016,Proceedings of SPIE--the International Society for Optical Engineering, pp.9786:97860A.

19.Banga V K, Singh Y, Kumar R, Uncertainty compensation and optimization of three and four degree-of-freedom robotics system using Genetic Algorithm, 2008, Modelling, Measurement and Control B,vol.77,no.1-2,pp.70-82.

20. Wang W, Zhang L, Ma J, Kinematic calibration of 6-UPS surgical parallel robot, 2013, ICME International Conference on Complex Medical Engineering, pp.369-374.

21. Ahola J M, SeppälÄ T, Koskinen J, et al, Calibration of the pose parameters between coupled 6-axis F/T sensors in robotics applications, 2017, Robotics \& Autonomous Systems, pp.1-8. 